\title{
Aplicación de un diseño curricular modular para la enseñanza del cálculo diferencial
}

\author{
Implementation of a modular curriculum for teaching differential calculus
}

\author{
Elías Irazoqui Becerra ${ }^{1} \quad$ Antonio Medina Rivilla ${ }^{2}$
}

Recibido 12 de abril de 2013, aceptado 20 de mayo de 2014

Received: April 12, $2013 \quad$ Accepted: May 20, 2014

\begin{abstract}
RESUMEN
El presente trabajo de investigación es el resultado de una serie de proyectos que se han desarrollado en la Universidad del Bío-Bío, Chile, encaminados todos ellos a mejorar el rendimiento académico de los estudiantes. Uno de dichos proyectos, en el que se sitúa esta investigación, comenzó el 2010, con un plan piloto para la carrera de Ingeniería Civil. La idea central de dicho proyecto fue diseñar los cursos de manera Modular, en tres módulos de trabajo. Entendiendo por módulo una parte del currículo original del curso. Ello significó una estructuración del curso en tres módulos para cubrir completamente los contenidos de la asignatura de Cálculo. Los buenos resultados académicos logrados por los estudiantes que cursaron la asignatura Modular de Cálculo Diferencial, no solo en esta carrera, han servido de base para realizar el presente estudio de investigación ahora en el contexto de la Pedagogía en Ciencias Naturales, rediseñando y evaluando tal propuesta Modular ahora en dos módulos de trabajo para su aplicación. Para ello se seleccionaron al azar dos grupos, uno experimental y otro control, el grupo experimental recibió la propuesta Modular, en cambio el grupo control no. La aplicación de este recurso metodológico de trabajo para el grupo experimental, unido a otras iniciativas que se comentan en este artículo, permitió un mejor rendimiento académico final por sobre los estudiantes del grupo control. Los resultados estadísticos obtenidos de tal experiencia educativa se encargan de demostrar tal hecho.
\end{abstract}

Palabras clave: Diseño curricular modular, enseñanza, cálculo diferencial.

\begin{abstract}
This research is the result of a series of projects that have been developed at Universidad del Bio-Bio, Chile, all aimed at improving the academic performance of students. One of such projects, in which this research is situated, began in 2010, with a pilot plan for the Career of Civil Engineering. The central idea of this project was to design in a modular way courses in three work modules. Module contain part of the original course curriculum. This required structuring the course in three modules in order to completely cover the contents of the Calculus course. Good academic results, achieved by students who completed the Modular Course of Differential Calculus, not only in this career, but also served as the basis for this research study in the context of Natural Sciences Pedagogy, redesigning and evaluating such a modular proposal of two modules for implementation. For the purpose, two randomly selected groups, Experimental and Control. The Experimental group received the Modular proposal, whereas the Control group did not. The application of this methodological work resource for the Experimental group, together with other initiatives discussed in this article, allowed for a better final academic performance than that of students on the Control group. This result is confirmed by means of statistical analysis.
\end{abstract}

Keywords: Modular curriculum design, teaching, differential calculus.

1 Ciencias Básicas. Universidad del Bío-Bío. Casilla 447. Chillán, Chile. E-mail: eirazoq@ubiobio.cl

2 Departamento de Didáctica. Organización Escolar y DD. Especiales. UNED. España. E-mail: amedina@edu.uned.es 


\section{INTRODUCCIÓN}

Las instituciones de educación superior, en general, realizan ingentes esfuerzos por mejorar la calidad de los aprendizajes de los estudiantes en todas sus materias de estudio; no ajeno a ello, la Universidad del Bío-Bío ${ }^{3}$ (Chile) ha venido desarrollando una serie de proyectos, todos ellos dentro del marco del mejoramiento de la calidad de la enseñanza superior impulsado por el Ministerio de Educación del país. Como resultado de esta política de Estado se han generado diversos proyectos que guardan relación con el quehacer docente, entre los que se pueden mencionar, atendiendo al orden del tiempo de su realización: "Innovación en el Proceso de EnseñanzaAprendizaje de la Carrera de Ingeniería Civil de la Universidad del Bío-Bío" (UBB 0604); "Diseño de Planes de Nivelación en Ciencias Básicas para el Primer Año de Ingeniería Civil" (UCO 0607) [1] $\mathrm{y}$, por último, el proyecto que ha dado sustento e inspiración al presente estudio de investigación, a saber: "Implementación de un plan de nivelación de competencias básicas y genéricas en ciencias básicas para alumnos de Ingeniería" (UBB 0809), este último proyecto se desarrolla desde el 2010 a la fecha. Entre sus objetivos principales está el mejorar las posibilidades de éxito académico de los estudiantes de primer y segundo año (ciclo inicial) de esta Universidad. El proceso de implementación involucra a la Facultad de Ciencias en aspectos multidimensionales, ya que se contempla el diagnóstico de habilidades y conocimientos de los alumnos que ingresan, y que cursan sus dos primeros años en esta Facultad, la reforma curricular de las asignaturas de Ciencias, la renovación metodológica de las prácticas docentes, el apoyo permanente a estos estudiantes, desde una acción de tutoría permanente y una gestión académica de aula ad hoc.

Este último proyecto a que se hace referencia, ha contado con el apoyo decidido de un proyecto institucional denominado Convenio de Desempeño, del que solo cuatro instituciones de educación superior pertenecientes al Consejo de Rectores (CRUCH) han participado. Todo ello ha permitido que la renovación curricular expresada en lo sustancial de una manera Modular al desarrollar las asignaturas, incremente el porcentaje de los alumnos que aprueban las asignaturas de Ciencias

3 http://www.ubb.cl
Básicas en Matemáticas y Física, disminuya el período de permanencia de los estudiantes en la universidad y, por lo demás, se logre con ello que el estudiante apruebe y domine los contenidos de cada Módulo de aprendizaje que conforma cada asignatura, pues de lo contario no logra la aprobación de la asignatura en cuestión. Por Módulo ha de entenderse una parte del currículum de la asignatura, así, en el caso del Cálculo Diferencial, ella se cubre completamente en dos Módulos de trabajo. El Módulo 1 se aboca al tratamiento de los conceptos de función, límite y continuidad de funciones y, por su parte, el Módulo 2 aborda el concepto de derivada y sus aplicaciones.

Todo lo anterior ha servido de marco referencial para el desarrollo de una propuesta didáctica ajustada a contexto, con el objetivo central de mejorar el rendimiento académico final de los estudiantes en la asignatura de Cálculo Diferencial, en un comienzo en la carrera de Ingeniería Civil, y posteriormente ir extendiendo esta propuesta que en lo sustancial se expresa en la realización Modular de la asignatura, a las otras carreras de Ingeniería que dicta la universidad y que, naturalmente, tienen al Cálculo Diferencial como una de sus materias. Ello implica además conjugar otros aspectos que se consideran importantes a la hora de impartirla, aprovechando en cada ocasión posterior la experiencia realizada con un fin de mejora, donde los docentes aprenden de la acción que realizan cada vez que el curso se imparte nuevamente [2].

Una breve síntesis de los aspectos esenciales de la propuesta que se ha implementado da cuenta de la consideración de aspectos como la evaluación continua de los aprendizajes, el uso de metodologías activas, como lo son la resolución de problemas, tanto rutinarios como no rutinarios; estos aspectos, junto a un acompañamiento tutorial al estudiante, han conformado el desarrollo de cada uno de los módulos, de modo de lograr aprendizajes efectivos en cada uno de los tópicos tratados por parte del profesor, cuando realiza la transposición didáctica en el aula.

Resulta oportuno señalar que si bien es cierto concurren durante la intervención metodológica otros aspectos como los señalados en el párrafo anterior, los resultados presentados en este estudio se centran en comparar dos metodologías de trabajo, 
una donde se usa un diseño curricular Modular y otra donde no se usa. Para ello se miden y se comparan estadísticamente ambas experiencias. Sin perjuicio de ello se tiene el convencimiento que este es un problema multidimensional, donde se combinan estrategias curriculares con estrategias didácticas.

\section{ANÁLISIS TEÓRICO}

La enseñanza de la Matemática para estudiantes de carreras no Matemáticas no puede ser la misma que para estudiantes que cultivan esta disciplina con miras a proseguir niveles mayores de profundidad en programas de Magister o Doctorado en Matemática. En el caso que nos ocupa, la formación de profesores de ciencias naturales -de Física, Química o Biología-, el adecuado conocimiento del Cálculo Diferencial resulta ser una herramienta que permite una mejor comprensión de las restantes materias propias de la disciplina, como son el Cálculo Integral y las Ecuaciones Diferenciales, por ejemplo. Pero, además, dichos temas matemáticos les permitirán adquirir una mejor comprensión de las otras disciplinas científicas propias de la formación a la que se adscriban, sea esta la Física, la Química o la Biología. Hay en lo anterior, entonces, un grado de responsabilidad que no solo atañe al estudiante, sino también al docente, y para este último la responsabilidad de generar más y mejores instancias de aprendizaje, es esta una tarea ineludible y tal vez nuestro único designio, si queremos que la tarea docente no pase de ser una más sin trascendencia ninguna para los educandos. De no asumir tal responsabilidad se hipoteca buena parte de la formación académica de los futuros maestros de ciencia del país, en las disciplinas de las ciencias básicas como Física, Química y Biología.

Por otro lado, muchas instituciones de educación superior, y esta en particular, están conscientes que sus estudiantes no alcanzan un aprendizaje con entendimiento, ello representa una problemática generalizada que alcanza a muchos países. Todo esto se traduce en que los estudiantes a pesar de aprobar los cursos de Cálculo solo aprenden rutinas, como es el caso de saber derivar, pero en lo fundamental no han comprendido las ideas que subyacen en el corazón del Cálculo, como lo es la comprensión del concepto de la Derivada, si se refiere al Cálculo Diferencial [3-4].
Ante estos hechos, los estudiantes pasan sus cursos de Cálculo pero no logran transferir el conocimiento del Cálculo Diferencial e Integral a las asignaturas que le siguen a esta, como son el Cálculo en Varias Variables y las Ecuaciones Diferenciales, por citar tan solo dos asignaturas posteriores que conforman, por lo general, el currículo de un buen número de las carreras en donde el conocimiento matemático es pieza fundamental.

Respecto del aprendizaje de los estudiantes, se ha asumido un enfoque sociocultural, donde los procesos de interacción constituyen algo consustancial a él. Se entiende que hacer matemáticas, y en este caso particular que el estudiante logre aprendizajes, se traduce en buena parte en la realización de actividades didácticas de aprendizajes que se han diseñado para tal propósito, de manera individual o grupal, constituyendo así la esencia de la construcción de su conocimiento matemático.

Bajo la postura anterior, donde el estudiante interactúa con su medio y sus pares, los instrumentos de mediación como son el software matemático, las propias actividades didácticas diseñadas para cada tópico a tratar se configuran como los verdaderos instrumentos de mediación de su aprendizaje al interactuar en su zona de desarrollo próximo [5].

Otro hecho no menos importante, digno de reportarse, es que la Matemática, y en particular el Cálculo, ha sido presentada a los estudiantes como un sistema conceptual lógico y estructurado, más acorde con el Análisis Matemático, aunque la Matemática en su esencia es eso. Lo anterior no amerita para que deba ser presentada de esta manera, donde los estudiantes se ven impedidos de recrear ciertas ideas que están en el corazón de ella, dejando así muy poca participación de hacer matemáticas descubriendo, haciendo conjeturas, probando dichas conjeturas. En resumen, resolviendo problemas que están a su alcance y donde la satisfacción de lograrlo sea un verdadero estímulo para continuar acrecentando su acervo matemático en formación.

En lo que se refiere al Cálculo Diferencial, se puede rescatar el estudio de la "razón de cambio" como un aspecto fundamental para aproximarnos al concepto de la Derivada, como ya han incursionado muchos investigadores en esta materia [6-7]. En este sentido, no hay que olvidar que la formalización de los conceptos 
matemáticos, por lo general, está precedida por las ideas intuitivas que dan origen a los mismos, prueba fehaciente de ello fue el concepto de límite, el que se formalizó y refinó a finales del siglo XIX con Cauchy y Weierstrass y, sin embargo, tanto Newton como Leibniz usaron muchas ideas matemáticas que en un comienzo no estaban claras ni para los propios inventores del Cálculo [8].

Sin perjuicio de lo ya acotado, es conveniente tener en cuenta a la hora de enseñar Cálculo, no suponer como verdaderos hechos como los siguientes:

\section{Un concepto se entiende con solo dar su definición} formal al estudiante; como sucede con la derivada, donde presentarla en términos de un límite ya basta para que sea comprendida a cabalidad, craso error. La experiencia indica que se necesita una variedad de ejemplos para acceder a una comprensión más profunda de este concepto, como podría ser mostrar distintos ejemplos de razones de cambio y de esta forma llegar de manera natural a su definición formal. Así, se transita de lo intuitivo a la formalización, como ha sido el desarrollo de la Matemática en el tiempo, ejemplos sobran en este sentido.

Comprenden un Teorema con solo darle la demostración lógica formal; este hecho en sí no es incorrecto, pero de ahí a suponer una comprensión de tal argumentación dista mucho, se reconoce sin duda el valor intrínseco de las demostraciones, pero no se está seguro de su total comprensión por parte de los estudiantes, en esto se debe reconocer que con esta postura se cometen bastantes errores entre los docentes.

Aplican las matemáticas en la resolución de problemas de manera adecuada; muchas veces hacen un uso incorrecto del conocimiento matemático, ya que este no se conoce a cabalidad y con ello no se puede alcanzar la solución del problema tratado.

Así, estas consideraciones, sumadas a la gran reprobación de las asignaturas de ciencias básicas, junto a los proyectos a que se han hecho mención justifican en buena medida la generación de una propuesta, desde su propio contexto, porque las instituciones al igual que las personas necesitan caminar por sí solas y darse su propio destino educativo en una búsqueda permanente de mejora, de modo de lograr resultados de aprendizajes óptimos en sus estudiantes.

No hay que olvidar que los procesos de enseñanza dependen en buena medida, por no decir totalmente, del entorno cultural y social en el que se desarrollan $y$, por ende, la validez de sus resultados es por tanto limitada, de manera que no se trata de acomodar cualquier paradigma educativo, sino más bien dar con el propio y ajustarlo al contexto en el que se desarrolla, solo así se estará logrando identificación y validez al proceso de enseñanza-aprendizaje entre sus actores principales, maestros y estudiantes [9].

Lo anterior no hace otra cosa que justificar una enseñanza del Cálculo Diferencial que esté al alcance del docente y, como es lógico, se pueda desarrollar in situ. Parafraseando a Lawrence Stenhause [10] quien afirma que ... solo el Profesor puede cambiar al Profesor, se puede afirmar entonces que: Solo la institución puede cambiar a la institución. De ahí que son las propias instituciones las que deben caminar hacia un cambio de renovación en sus prácticas educativas $\mathrm{y}$, con ello, conformar un nuevo paradigma si desean realizar progresos en sus procesos de enseñanza, buscando nuevas y mejores metodologías de trabajo a nivel de aula para lograr avances en su desempeño educacional, sean estos en materia de contenidos disciplinares, respecto de qué enseñar y cómo hacerlo, como también suplir las falencias en materia de formación integral en su sentido más amplio posible y, de esta manera, formar profesionales más capaces y competentes, con ello se estará, en alguna medida, cumpliendo con la misión y visión que la institución se ha dado a sí misma en materia docente.

Naturalmente que conformar un nuevo paradigma en materia de enseñanza y aprendizaje pasa por darse cuenta de la necesidad de ello, como fruto de la "reflexión académica en materia de enseñanza" y, además, estar atentos a los signos y señales del entorno en que hoy se está inmerso, donde las competencias y habilidades del futuro profesional deben ser consideradas por los formadores de las casas de estudios superiores.

En consideración a lo anterior, se ha puesto el énfasis tanto en el diseño como en la implementación de Actividades Didácticas de Aprendizaje (ADA) en las que se han involucrado temas como: 
un aprendizaje basado en problemas, que incluye la resolución de problemas tanto rutinarios como no rutinarios, y en donde los problemas abordados no se limitan al contexto matemático sino a las demás Ciencias Naturales (Física, Química o Biología) [11].

No priorizar el lenguaje formal del contenido matemático, aunque esta es una tarea difícil, porque por lo general los problemas y, por ende, su resolución se realizan bajo este lenguaje. Lo ideal sería avanzar en la formalización como consecuencia de la propia actividad matemática de los estudiantes con los problemas que abordan y, como consecuencia, aprecien la necesidad de una formalización matemática mayor para expresar sus resultados.

El uso de diversas representaciones de un concepto es otra idea que contribuye al aprendizaje, como asî mismo el cambio de registro de una representación a otra, como ocurre con el caso de las funciones y en la introducción del concepto de la derivada, por ejemplo [12].

La disponibilidad de los recursos informáticos ha permitido la ayuda tanto como un soporte de mediación semiótica como en la visualización de los conceptos matemáticos a la hora de diseñar las actividades didácticas de aprendizaje. En concordancia con ello, se han privilegiado los recursos informáticos de dominio público, unido al uso de la plataforma virtual que la institución utiliza para poner en la red los diversos recursos didácticos que sirven de apoyo para el aprendizaje del Cálculo [13-14].

Otros aspectos importantes que se suman a los ya expuestos y que no guardan tan estrecha realización con las ADA, pero que se suman al esfuerzo común de un aprendizaje de mayor calidad, dicen relación con aspectos como:

La evaluación continua, que obliga al estudiante a una revisión permanente de las materias tratadas, pues los contenidos se suceden rápidamente y el tiempo no permite detenerse mucho en una temática específica, de lo contrario se corre el riesgo de no cubrir todos los acápites que conforman el currículo del curso de Cálculo. Luego, como una forma de cerciorarse que se están logrando los aprendizajes con entendimiento por parte de los estudiantes, se ha optado por la modalidad de una evaluación continua, resistida en un comienzo por los estudiantes, pero que poco a poco ha dado sus frutos.

La asignación de tareas y pequeñas investigaciones de manera grupal ha contribuido a la integración social del grupo curso. Hoy casi no se concibe un trabajo que no sea en equipo y, por tanto, preparar a los estudiantes en esta modalidad de trabajo los acerca de manera natural a su futuro mundo laboral que deberán enfrentar ad portas [15].

Por último, todos estos aspectos han sido conjugados bajo la perspectiva del Modelo Educativo que esta universidad trata de implementar en materia docente, en donde el protagonista central de su aprendizaje es el estudiante, y tanto el cuerpo de profesores como la administración universitaria solo le sirven de apoyo. Esto que en el papel resulta fácil, no lo es tanto en la práctica diaria del aula. Los estudiantes han sido educados para que sea el profesor quien desarrolle la clase de punta a cabo y si él no está no hay clases. Cambiar este paradigma no será fácil y hacerlo responsable de su aprendizaje menos aún. Se debe además hacer entender a los estudiantes que lo que ellos realizan es muy importante a la hora de aprender y que su proceso formativo recién comienza, y el aprendizaje ha de ser un continuo a lo largo de la vida [16-18].

\section{DISEÑO E IMPLEMENTACIÓN}

Esta propuesta didáctica en contexto ha sido desde su inicio, 2010 a la fecha, la implementación de la renovación curricular de las asignaturas basadas en competencias, y en donde además las asignaturas tanto de Matemáticas como de Física de los primeros años de la carrera de Ingeniería Civil de esta universidad han usado como eje central de dicha propuesta este recurso metodológico Modular para desarrollar cada una de las asignaturas. Esta idea de -en cierto modo- dividir la asignatura no es nueva, otras instituciones con más trayectoria han usado este recurso metodológico para materializar su currículum, como ha sido el caso de la Universidad Tecnológica del Paso, Texas ${ }^{4}$ (UTEP).

Es claro además que conformar una nueva propuesta de trabajo docente para el aula pasa por puntos

4 http://www.utep.edu 
esenciales, como pueden ser: mostrar ejemplos exitosos de que tal propuesta puede y ha sido exitosa en otros lugares con estudiantes de características similares a ellos, en segundo lugar convencer a los docentes que tal propuesta puede tener éxito y capacitarlos en el uso de metodologías activas de trabajo donde el estudiante pasa a ser el protagonista principal de su aprendizaje y, por último, tener los recursos necesarios para implementar tal propuesta. Sin estas observaciones que ahora pasan de perogrullo, no se habría realizado tal experiencia educativa, por muy simples que parezcan, porque han significado mucho trabajo y esfuerzo para obtener lo que ahora se puede exhibir en materia de rendimiento académico logrado por los estudiantes, ello sin duda representa un claro aliciente que llena de satisfacción.

Reiteramos que se entiende por Módulo una unidad curricular de una duración de tiempo necesaria y suficiente para desarrollar una parte de los contenidos de una asignatura semestral. Así, en un comienzo, hechos los ajustes necesarios, cada asignatura del antiguo plan de estudio quedó conformada en tres Módulos, denominados Módulo 1, Módulo 2 y Módulo 3.

La aprobación de la asignatura por parte del estudiante queda ahora sujeta a la aprobación de cada uno de los Módulos, y su calificación final resulta ser el promedio aritmético de la nota que obtuvo en cada Módulo. La no aprobación del Módulo 1 obliga al estudiante a repetir dicho Módulo, sin tener acceso al Módulo 2; si vuelve a reprobar el Módulo 1 debe en el transcurso del desarrollo del Módulo 3, del cual no participa, cursar por última vez el Módulo 1; si reprueba nuevamente deberá cursar la asignatura por segunda oportunidad. La aprobación del Módulo 1 faculta para cursar el Módulo 2, y así siguiendo, hasta completar los tres Módulos del curso. Esta nueva forma de estructurar las asignaturas de Matemáticas y Física, si bien es cierto redunda en más trabajo docente, ha logrado mejorar los rendimientos académicos de los estudiantes en las asignaturas modulares.

Por otro lado, el mayor costo que ello ha significado tanto en tiempo como en recursos humanos de parte de la universidad se ha visto favorecido por un mayor índice de aprobación de los estudiantes que han cursado las asignaturas modulares.
La propia experiencia y estas observaciones de índole económico ha obligado a rebajar la cantidad de Módulos de tres (3) a dos (2), desde el 2011 a la fecha, con resultados óptimos de aprobación de las asignaturas, si se los compara con los que se venían dando en promedio antes que esta propuesta metodológica se materializara, como podrá apreciarse al momento de examinar las estadísticas del presente estudio de investigación.

Ahora, en lo que dice relación específicamente con el aprendizaje del Cálculo Diferencial, y en relación con los aspectos teóricos de referencia aludidos, se puede señalar que la enseñanza del Cálculo Diferencial se ha implementado en dos Módulos, a saber:

En el primero se abordaron las temáticas de funciones, límite y continuidad, estos dos últimos temas se abordaron desde una postura intuitiva y como aproximación, usando las diferentes posibilidades que ofrece el concepto de función en materia de representación, lo que ha ayudado a una mejor comprensión de estos conceptos matemáticos, como han sido las indicaciones de múltiples investigadores que se han ocupado de estos temas [19].

Por su parte, el segundo Módulo contempló las temáticas del Concepto de Derivada, el Álgebra de Derivadas y sus Aplicaciones. De estos tres temas la mayor cantidad de estudios de investigación a nivel mundial se centran en el "Concepto de la Derivada", dando a entender con ello que tanto el álgebra de derivadas como su consecuente aplicación será el resultado de una buena comprensión del primero.

Siguiendo la idea anterior se ha invertido mayor tiempo en el primer tema, usando la mayor cantidad posible de ejemplos donde la razón de cambio es la idea central. Afianzar y volver a examinar esta postura, que guarda relación ahora con la forma en la que se hace la transposición didáctica, dará la confianza necesaria para conformar un paradigma educativo propio para el aprendizaje del Cálculo Diferencial ajustado al contexto educativo particular y, por tanto, con la necesaria validez que este estudio ha dado en el tiempo, donde paulatinamente se ha ido aprendiendo de los propios errores a la hora de enseñar el Cálculo Diferencial de una variable [20]. 
En lo que se refiere a textos de Matemáticas consultados están los habituales: Lang, Larson en su octava edición, Purcell, Stewart en su cuarta edición y Hughes-Hallett en su segunda edición. Además de estos textos de consulta los estudiantes dispusieron de apuntes como apoyo a sus clases presenciales [21-24].

Se ha mencionado previamente el hecho que tanto las actividades didácticas como los contenidos propiamente tal que comprende el Cálculo Diferencial han sido dispuestos en la plataforma virtual Adecca de que dispone la institución, y una próxima apuesta será usar la plataforma virtual Moodle [26]. Esto, sin duda, obligará a hacer evaluaciones respecto de ambos entornos virtuales, de modo de optar por aquella que ofrezca mejores prestaciones y sea mejor evaluada por los estudiantes; de paso se puede agregar que Facebook se constituye, también, en un excelente medio de comunicación tanto entre los estudiantes como cuando se desea dejar un mensaje o un Link de interés que complementa la materia que se está tratando, este hecho aunque parezca intrascendente posee mucho valor, pues en definitiva son los usuarios de dicha red los que le dan sentido y vida a esta comunidad virtual hoy en boga.

Lo que se acaba de afirmar se ha constatado experimentalmente y se configura como un hecho completamente normal y propio del comienzo de este siglo XXI, donde el uso de las redes sociales cobra cada día mayor importancia en el plano docente.

\section{EVALUACIÓN}

El primer semestre del 2010 se dio comienzo a este estudio de innovación con la metodología Modular para impartir los cursos tanto de Matemáticas como de Física en el primer año de la carrera de Ingeniería Civil de esta casa de estudios. Esta primera experiencia de carácter piloto estableció las bases sobre las cuales se podrían construir los cursos posteriores. Los cursos que usaron esta estrategia metodológica en un comienzo fueron, aparte del Cálculo Diferencial: Álgebra y Trigonometría, Cálculo II (Cálculo Integral), Álgebra Lineal y en el segundo semestre de ese mismo año la asignatura de Física I.

La tabla siguiente resume estadísticamente los resultados obtenidos el 2010, versus el promedio entre los años 2005 al 2009, en la carrera de Ingeniería Civil, en cinco asignaturas, donde se usó la modularización como estrategia didáctica para su realización.

Tabla 1. \% aprob. promedio asig. 2005-2009 versus \% aprob. asig. modulares 2010.

\begin{tabular}{|l|c|c|}
\hline \multicolumn{1}{|c|}{ Asig. } & $\begin{array}{c}\text { \% de } \\
\text { aprobación } \\
\text { promedio } \\
\mathbf{2 0 0 5 - 2 0 0 9}\end{array}$ & $\begin{array}{c}\text { \% de aprobación } \\
\text { asignaturas } \\
\text { modulares } \\
\mathbf{2 0 1 0 .}\end{array}$ \\
\hline $\begin{array}{l}\text { Álgebra } \\
\text { y Trig. }\end{array}$ & $59,6 \%$ & $96 \%$ \\
\hline $\begin{array}{l}\text { Álgebra } \\
\text { Lineal }\end{array}$ & $76,2 \%$ & $97,4 \%$ \\
\hline Cálculo I & $\mathbf{4 7 , 1 \%}$ & $\mathbf{8 7 , 6 5 \%}$ \\
\hline $\begin{array}{l}\text { Cálculo } \\
\text { II }\end{array}$ & $62,8 \%$ & $65 \%$ \\
\hline Física I & - & $86 \%$ \\
\hline
\end{tabular}

Fuente: http://convenio.ubiobio.cl/capsulas/ modularizacion.html

Durante el 2011 se extiende esta experiencia Modular a carreras afines a la Ingeniería Civil, como son las carreras de Ingeniería Civil Industrial e Ingeniería Civil Mecánica. Los porcentajes de aprobación de la asignatura de Cálculo I vuelven a situarse en torno al $80 \%$ en promedio, con una variación mínima entre ellas.

Amparados en estos buenos resultados de aprendizajes logrados por los estudiantes, que se han traducido en un aumento significativo en los niveles de aprobación no tan solo en la asignatura de Cálculo 1, motivaron la realización de una nueva experiencia de innovación mediante la estrategia didáctica Modular en la carrera de Pedagogía en Ciencias Naturales en el campus Chillán, usando para ello un grupo control, de modo de poder medir y contrastar qué tan significativa era esta propuesta de trabajo al impartir dicho curso en otra carrera que no fuese una Ingeniería, como hasta ahora había ocurrido.

Así, la población de interés estuvo constituida por 56 estudiantes de la mencionada carrera (Pedagogía en Ciencias Naturales), la que fue dividida al azar en dos grupos de 28 estudiantes cada uno, los que constituyeron tanto el grupo experimental como el grupo control. Los estudiantes que conformaron el grupo experimental llevaron el curso de Cálculo 
bajo esta nueva propuesta didáctica basada en la modularización, por su parte el grupo control, como es obvio, recibió el curso de la forma regular. Conviene tener presente que en un comienzo, año 2010, se usaron tres Módulos para impartir cada uno de los cursos que usaron la estrategia Modular.

Los resultados de la experiencia pedagógica que a continuación se relata están basados en solo dos Módulos, ello también ha sido el consenso de un aprendizaje de la realización de la propia experiencia. Con ello se hace patente lo útil y necesario que resulta que las instituciones aprendan de sí mismas $y$, por tanto, la validez de sus propuestas en contexto adquieren una notable trascendencia para ellas.

Los resultados estadísticos de carácter cuantitativo de este estudio se resumen en los siguientes hechos:

Para el grupo experimental: aprobación del Módulo 1: 24 estudiantes con $86 \%$ de aprobación. Reprobación del Módulo 1: 4 estudiantes con 16\% de reprobación. En la repetición del Módulo 1: 0\% de aprobación. En la dictación del Módulo 2: 100\% de aprobación, esto es, los 24 estudiantes aprobaron el Módulo 2. En resumen, el grupo experimental obtuvo $86 \%$ de aprobación.

El grupo control por su parte obtuvo $54 \%$ de aprobación, es decir, 15 estudiantes aprobaron la asignatura y $46 \%$ la reprobaron, lo que se traduce en que 13 estudiantes reprobaron la asignatura.

Los Test R de Rachas y el Test U de Mann-Whitney, con los que se realiza el estudio cuantitativo se usaron por dos razones: uno, el tamaño pequeño de la muestra $(n=28) y$, segundo, no se necesita de la hipótesis de normalidad.

Luego, si se comparan las notas finales obtenidas por ambos grupos y se usa la estadística que se condice con esta experiencia de aula, bajo las condiciones descritas anteriormente, se puede señalar que: usando el Test $\mathrm{R}$ de Rachas se obtuvo los siguientes valores, los cuales se presentan en la Tabla 2.

Para un nivel de significación del 5\% no se rechaza la hipótesis nula $\mathrm{H}_{0}$ de aleatoriedad, ya que el valor-p $=0,906>0,05$ y se concluye que los datos constituyen una muestra aleatoria.
Tabla 2. Pruebas de rachas de aleatoriedad.

\begin{tabular}{|l|c|}
\hline \multicolumn{1}{|c|}{ Test de Rachas } & Nota final \\
\hline Valor de prueba & 4,2 \\
\hline Casos < Valor de prueba & 24 \\
\hline Casos >= Valor de prueba & 32 \\
\hline Casos en total & 56 \\
\hline Número de rachas & 28 \\
\hline Z & $-0,118$ \\
\hline Sig. asintót. (bilateral) & 0,906 \\
\hline
\end{tabular}

Usando el Test U de Mann-Whitney se obtuvieron los siguientes valores: por grupo: control y experimental, dichos valores están consignados en la Tabla 3.

Tabla 3. Prueba U de Mann-Whitney entre Grupo Control y Grupo Experimental.

\begin{tabular}{|l|c|}
\hline Test U de Mann-Whitney & Nota final \\
\hline U de Mann-Whitney & 139,000 \\
\hline W de Wilcoxon & 545,000 \\
\hline Z & $-4,155$ \\
\hline Sig. asintót. (bilateral) & 0,000 \\
\hline
\end{tabular}

El análisis arroja diferencias estadísticamente significativas en los promedios finales para el grupo experimental y el de control $(-\mathrm{p}<0,001)$.

El grupo experimental registra un promedio superior al grupo control, con un 4,7 y 3,7 respectivamente. Por género:

Tabla 4. Prueba U de Mann-Whitney entre género.

\begin{tabular}{|l|c|c|}
\hline $\begin{array}{c}\text { Test U de } \\
\text { Mann-Whitney }\end{array}$ & $\begin{array}{c}\text { Promedio } \\
\text { final grupo } \\
\text { control }\end{array}$ & $\begin{array}{c}\text { Promedio } \\
\text { final grupo } \\
\text { experimental }\end{array}$ \\
\hline $\begin{array}{l}\text { U de Mann- } \\
\text { Whitney }\end{array}$ & 85,5 & 36 \\
\hline W de Wilcoxon & 140,5 & 42 \\
\hline Z & $-0,217$ & $-0,112$ \\
\hline $\begin{array}{l}\text { Sig. asintót. } \\
\text { (bilateral) }\end{array}$ & 0,828 & 0,911 \\
\hline
\end{tabular}

De la Tabla 4 se concluye que no hay diferencias estadísticamente significativas entre los promedios finales por género para los dos grupos objeto de estudio (valor $-\mathrm{p}>0,1$ ). 


\section{CONCLUSIONES}

Los resultados estadísticos anteriormente presentados permiten concluir que hay evidencias para asegurar que los alumnos que desarrollaron su proceso de aprendizaje bajo la estructura modular obtuvieron, en promedio, mejores calificaciones que aquellos sometidos a la metodología tradicional y que constituyeron el Grupo Control.

Bajo este recurso metodológico Modular han intervenido una serie de otros elementos, que de manera conjunta en el desarrollo del curso hicieron posible tales resultados. Sin embargo, conviene hacer notar que esta nueva metodología ha sido clara y decidora como política docente. Aún se está lejos de afirmar que la modularización y todo lo que ello ha involucrado en el cuerpo docente y estudiantil será la panacea para la enseñanza del Cálculo, bien es sabido que educar es un arte y esta es una propuesta más que se agrega a la lista de recursos metodológicos para obtener mejores resultados de aprendizaje en los estudiantes, que poco a poco comienza a rendir su frutos y comienza a instalarse en las asignaturas de primer año para los estudiantes.

Junto a lo anterior, se suma un sinnúmero de iniciativas que de manera conjunta pueden explicar tal éxito, entre ellas se pueden mencionar: el proceso de evaluación continua a que fueron sometidos los estudiantes, ello los obligó a estar atentos a sus materias y a no jugar con los promedios, para aprobar su asignatura, pues si no aprueban el Módulo 1 no pueden continuar con el Módulo 2. Además, ahora los estudiantes tienen otra posibilidad, al repetir el Módulo que reprueban. Está también el trabajo práctico con las actividades didácticas realizadas de manera grupal y/o individual, en las que fueron apoyados por el profesor y el ayudante, es este otro elemento a considerar en el buen rendimiento final alcanzado por los estudiantes.

Respecto de los contenidos disciplinares tratados, dispusieron de abundante material en el espacio virtual consignado para ello en la plataforma virtual Adecca, administrada por el profesor y el ayudante. Agregar además que los contenidos se abordaron desde un comienzo de manera intuitiva, para posteriormente avanzar a su formalización matemática. Para ello se usaron diversas representaciones, con lo que la aprehensión del conocimiento se facilita, como bien se sabe.

Aunque no se expuso anteriormente, se aplicó una encuesta a los estudiantes sobre la metodología usada, la respuesta a dicha encuesta arrojó un alto grado de satisfacción, cercana al cien por ciento respecto de la forma en la que se impartió la asignatura. Esto es otro elemento digno de destacarse, en una sociedad marcada por la calidad de los servicios académicos que se prestan hoy.

Por último, conviene aclarar que la exposición de este artículo ha centrado su atención preferentemente en el recurso metodológico Modular, comparando su uso entre un grupo control que lo usó y otro que estuvo desprovisto de él. Se está consciente que dicho recurso por sí solo no explica completamente el mejor rendimiento académico hasta ahora obtenido por los estudiantes, pero lo importante ha sido que su implementación ha favorecido la participación de otras iniciativas que actuando de manera conjunta ha permitido obtener los rendimientos académicos de los estudiantes en niveles superiores a los que se tenían, en ello sí que hay una gran conclusión a tener en cuenta.

\section{PROYECCIONES DEL ESTUDIO}

A partir de lo ya realizado, nuevas asignaturas de la carrera de Pedagogía en Ciencias de la Universidad (UBB) se sumarán al Cálculo Diferencial, usando como recurso didáctico fundamental la modularización para ser impartidas, entre ellas estarán: Química I y Química II, Introducción a la Física e Introducción a la Matemática, esta última previa al Cálculo Diferencial, todas estas asignaturas corresponden al primer año de su plan de estudio. La elección de dichas asignaturas se ha basado principalmente en dos hechos fundamentales: el bajo rendimiento académico de los estudiantes, hecho que se ha venido reportando desde hace varios años y, en segundo lugar, los resultados positivos obtenidos al usar la modularización no solo en la asignatura de Cálculo Diferencial, como ha quedado de manifiesto.

Lo señalado anteriormente no hace otra cosa que proyectar este trabajo a las otras disciplinas científicas, como son la Física y la Química, de modo tal que los profesores de estas disciplinas 
incursionen en la enseñanza de las mismas con nuevas propuestas didácticas innovadoras, y en donde la modularización les sirva de pilar fundamental.

Por último, los estudios de investigación por lo general se inspiran y sustentan en proyectos de mayor envergadura, tal ha sido el caso del presente estudio, donde tanto el proyecto Mecesup 0809 y el proyecto institucional Convenio de Desempeño, han sido las bases que han permitido la ejecución del presente estudio. El proyecto institucional aludido ha tenido entre una de sus finalidades principales "Disminuir significativamente la deserción de los estudiantes de la Universidad del Bío-Bío, con especial énfasis en los alumnos de grupos social y económicamente vulnerables".

No cabe la menor duda que a la luz de los resultados hasta ahora obtenidos la modularización y todo lo que ella comporta como recurso metodológico nuevo para esta casa de estudios se constituye en una posibilidad cierta de mejorar los resultados de aprendizajes de los estudiantes. Ello implica necesariamente una revisión permanente de las asignaturas que se dicten bajo esta modalidad de trabajo, de modo de aprender de los errores cometidos en pos de una mejora y por el bien de sus futuros estudiantes.

\section{AGRADECIMIENTOS}

Agradecemos al Ministerio de Educación y a los docentes que participaron de esta iniciativa de innovación didáctica, con el noble propósito de mejorar los procesos formativos de nuestros estudiantes que dan sus primeros pasos en su vida universitaria en las ciencias básicas.

\section{REFERENCIAS}

[1] "Proyectos Mecesup UBB-0809". Universidad del Bío-Bío. Fecha de consulta: 12 de diciembre de 2012. URL: http://mecesup. ubiobio.cl/proyectos_en_curso3.html

[2] A. Latorre. "La investigación-acción: Conocer y cambiar la práctica educativa". Editorial GRAÓ. Barcelona, España. 2003. ISBN: 84-7827-292-5.

[3] A. Engler, S. Vrancken y D. Müller. "Las derivadas: actividades que favorecen su comprensión". Revista Novedades Educativas. Año $15 \mathrm{~N}^{\circ}$ 146, pp. 32-36. Febrero de 2003.

[4] A. Engler, S. Vrancken y D. Müller. "La variación y el cambio: tópicos para el desarrollo del pensamiento matemático". Fecha de consulta: 10 de abril de 2012. URL: http://www.unam.edu.ar/

[5] F. Barriga y G. Hernández. "Estrategias docentes para un aprendizaje significativo". McGraw-Hill. México. 1999.

[6] E. Wenzelburger. "Cálculo Diferencial, una guía para maestros y alumnos". Grupo Editorial Iberoamérica, S.A. México. 1993. ISBN: 968-7270-66-7.

[7] R. Cantoral. "Hacia una didáctica del Cálculo basada en la cognición". Publicaciones Centroamericanas. Vol. 7, pp. 391-410. 1993.

[8] A. Duran. "La verdad está en el límite". Barcelona: RBA Libros, S.A. 2011. ISBN: 978-84-9867-945-8.

[9] M. Artigue. "La Enseñanza de los principios del cálculo: problemas epistemológicos, cognitivos y didácticos". Ingeniería Didáctica en Educación Matemática. Grupo Editorial Iberoamérica. México. 1995.

[10] L. Stenhouse. "La investigación como base de la enseñanza". Ediciones Morata. Madrid, España. 2004. ISBN: 84-7112-315-0.

[11] L. García, M. Moreno y C. Azcárate. "EBP como metodología activa para la enseñanza del Cálculo Diferencial". Fecha de consulta: 23 de agosto de 2012. URL: www.saber. ula.ve/bitstream/123456789/16638/1/ ebp_metodologia.pdf

[12] R. Duval. "Representation, Vision and Visualization: Cognitive Functions in Mathematical Thinking. Basic Issues for Learning". Proceeding of the Meeting of the North American Chapter of the International Group for the Psychology Mathematics Education. Date of visit: April 12, 2011. URL: http://patthompson.net/ PDFversions/1999Duval.pdf

[13] L. Trouche. "Recursos para procesar, aprender, enseñar el cálculo: nuevos modos de concepción y difusión". Fecha de consulta: 20 de mayo de 2012. URL: http:// educmath.ens-lyon.fr/Educmath/recherche/ approche_documentaire/1trouche09saltillo. pdf 
[14] J. Monje. "Visualización del conocimiento en la enseñanza-aprendizaje del Cálculo Diferencial". Fecha de consulta: 10 de enero de 2013. URL: http://www.cimm.ucr. ac.cr/ ocs/index.php/xiii_ciaem/xiii_ciaem/paper/ viewFile/2497/798

[15] H. Cofré, J. Camacho, A. Galaz, J. Jiménez, D. Santibáñez y C. Vergara. "La educación científica en Chile: debilidades de la enseñanza y futuros desafíos de la educación de Profesores de ciencia". Estudios Pedagógicos. XXXVI No 2, pp. 279-293. 2010. ISSN: 0718-0705.

[16] H. Guínez. "Formación del profesorado de matemáticas mediante el diseño de actividades innovadoras". Tesis para optar al grado de Doctor (no publicada). UNED, España. 2011.

[17] J. Biggs. "Calidad del aprendizaje universitario”. Madrid: Narcea, S.A. 2010. ISBN: 978-84-277-1398-7.

[18] "Orientaciones para la implementación del Modelo en el marco de la Renovación Curricular en la Universidad del Bío-Bío". Documento $\mathrm{N}^{\circ} 1$. Vicerrectoría Académica. 2010.

[19] D. Gil Pérez y M. de Guzmán Ozámiz. "La Enseñanza de las Ciencias y la Matemática". Editorial Popular. Madrid, España. 2001. ISBN: 84-7884-243-8.
[20] J.R. Jiménez. "La naturaleza global y dinámica de la derivada como objeto matemático y de su enseñanza". Fecha de consulta: 5 de noviembre de 2012. URL: http://semana. mat.uson.mx/MemoriasXVII/XIII/indice. htm

[21] S. Lang. "Cálculo". Addison-Wesley Iberoamericana. S.A. México. 1990. ISBN: 0-201-62906-2.

[22] R. Larson, R. Hostetler y B. Edwards. "Cálculo I". México. McGraw-Hill / Interamericana. Editores, S.A. de C.B. 2006. ISBN: 0-618-50298-X.

[23] E. Purcell y D. Varberg. "Cálculo diferencial e integral”. México. Prentice-Hall. 2000. IBSN: 970-17-0413-4. Serie AWLI.

[24] J. Stewart. "Cálculo de una variable". México. Cengage Learning. 2010. ISBN-13: 978-607-481-237-4.

[25] D. Hughes-Hallett, A. Gleason, P. Frazer y D. Flatth. "Cálculo Aplicado". México. Grupo editorial patria, S.A. de C.V. 2008. ISBN: 978-970-24-0725-6.

[26] J. Sánchez, P. Sánchez, y F. Ramos. "Usos pedagógicos de Moodle en la docencia universitaria desde la perspectiva de los estudiantes". Revista Iberoamericana de Educación. No 60, pp. 15-38. 2012. 